\section{Tuberculosis During Covid-19 Pandemic: Challenges and Opportunities}

Both novel severe acute respiratory syndrome coronavirus 2 (SARS-CoV-2) and Mycobacterium tuberculosis involve the lungs and share symptoms like cough, fever, and respiratory difficulty. The transmission for both agents is through aerosol and close contact, although the incubation period for tuberculosis is relatively longer. There is limited experience of COVID-19 infection in tuberculosis (TB) patients, but it is estimated that there may be a catastrophic impact of COVID19 on TB [1]. This pandemic of COVID-19 has elicited social stigmata and discriminatory behaviour, coupled with preexisted TB related stigmata [2,3]. Together, it is likely to increase the burden on the family as well as on limited resources in developing countries.

Currently, GeneXpert is the primary diagnostic tool for TB; however, its equipment is shifted and being used for COVID-19 testing, which is seriously jeopardizingthe testing capacities for TB [4]. Moreover, the protection of health care staff involved in the TB program is a big concern, as sputum production is considered as an aerosol generating procedure [5]. Further-more, access to health care facilities is also hampered due to lockdown. This may lead to interruption of treatment and increase the possibility of drug resistance TB (DR-TB). All stakeholders should ensure that gene expert machines during the COVID-19 pandemic should be used in such a manner that there should be fractional testing for both COVID-19 and TB. Health care workers (HCW) and other personnel involved in TB care should be retrained on the importance of universal safety precautions and infection prevention control. The mechanism of the door to door drug delivery needs to be developed and strengthened in such an extent that the treatment of TB will not be hampered. The universal use of masks in COVID-19 will also help in reducing the incidence of TB, and considering the high TB burden in India, this practice should be continued in the post-COVID era too, especially in crowded areas.

We feel that lessons learned from this pandemic can be fruitfully be used for tuberculosis eradication in future.

Published Online: September 05, 2020; PII:S097475591600241

Prawin Kumar and Jagdish P Goyal* Department of Pediatrics, All India Institute of Medical Sciences, Jodhpur, Rajasthan, India.

\section{REFERENCES} *jpgoyal@rediffmail.com

1. Boffa J, Mhlaba T, Sulis G, et al. COVID-19 and tuberculosis in South Africa: A dangerous combination. S Afr Med J. 110:2020. Published online 2020 March 27.

2. Alagna R, Besozzi G, Codecasa LR, et al. Celebrating World Tuberculosis Day at the time of COVID-19. Eur Respir J. 2020;55:2000650.

3. Adepoju P. Tuberculosis and HIV responses threatened by COVID-19 [published online ahead of print, 2020 Apr 8]. Lancet HIV.

4. World health Organization (WHO) Information Note onTuberculosis and COVID-19. Accessed May 6, 2020.

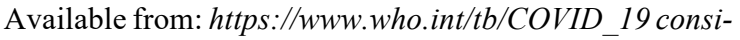
derations_tuberculosis_services.pdf

5. Tuberculosis and Covid 19. Accessed May 23, 2020. Available from: https://www.who.int/docs/default-source/ documents/tuberculosis/infonote-tb-covid-19.pdf

\section{Pediatric Renovascular Hypertension: The Diagnostic Algorithm}

Apropos of the recently published review on management of renal artery stenosis in the journal [1]. Diagnostic algorithm in the article shows that diagnosis is to be confirmed by Digital Subtraction Angiogram (DSA) even if findings are suggestive of renal artery stenosis (RAS) on computed tomograhy angiography (CTA) or magnetic resonance angiography (MRA). In this context it is prudent to note that MRA is reported to have a sensitivity of $62.5 \%$ for RAS detection with $100 \%$ specificity [2], whereas sensitivity for CTA is known to be as high as $84.2 \%$ [2]. Authors have rightly pointed out radiation risks associated with CTA and DSA. In pediatric diagnostic imaging modalities, efforts are always made to reduce the radiation exposure by using radiation reduction protocols $[3,4]$. DSA unarguably remains gold standard for accurate diagnosis of RAS with a sensitivity and specificity of almost $100 \%$ [2]. However, it is the most invasive of all tests, requires anesthesia and involves radiation exposure. Thus, if CTA or MRA findings are suggestive of RAS then there should not be any need for DSA for diagnosis. 